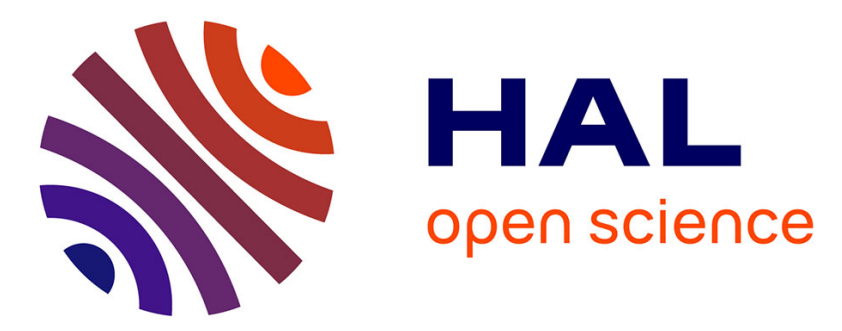

\title{
Exponential stabilization of a clamped Timoshenko beam with actuation on a tip mass
}

\author{
Andrea Mattioni, Yongxin Wu, Yann Le Gorrec
}

\section{To cite this version:}

Andrea Mattioni, Yongxin Wu, Yann Le Gorrec. Exponential stabilization of a clamped Timoshenko beam with actuation on a tip mass. 60th IEEE Conference on Decision and Control (CDC 2021), Dec 2021, Austin, United States. hal-03549324

\section{HAL Id: hal-03549324 \\ https://hal.science/hal-03549324}

Submitted on 31 Jan 2022

HAL is a multi-disciplinary open access archive for the deposit and dissemination of scientific research documents, whether they are published or not. The documents may come from teaching and research institutions in France or abroad, or from public or private research centers.
L'archive ouverte pluridisciplinaire HAL, est destinée au dépôt et à la diffusion de documents scientifiques de niveau recherche, publiés ou non, émanant des établissements d'enseignement et de recherche français ou étrangers, des laboratoires publics ou privés. 


\title{
Exponential stabilization of clamped Timoshenko beam with a tip mass
}

\author{
Andrea Mattioni ${ }^{1}$ and Yongxin $\mathrm{Wu}^{1}$ and Yann Le Gorrec ${ }^{1}$
}

\begin{abstract}
In this paper we consider the stabilization problem of a clamped beam with torque and force actuation on a mass in the other side of the beam. We show how to derive the model starting from the Principle of Least Action and we rewrite it as the interconnection between a 1 dimensional distributed parameter port-Hamiltonian system and a finite dimensional port-Hamiltonian system. Therefore, we propose a control law that allow to exponentially stabilise the origin of the closedloop system. In this preliminary paper we only sketch the theoretical proofs, but we give the procedure to compute the exponential bound of the system's state. Finally, we provide some numerical simulations testing the closed-loop behaviour with different choices of the control parameters.
\end{abstract}

\section{INTRODUCTION}

Systems modelled by a mixed set of Partial Differential Equations (PDE) and Ordinary Differential Equations (ODE) have a wide range of applications, ranging from spatial manipulators [1] to micro-grippers [2]. This is because distributed parameters systems modelled by a set of PDE are frequently controlled by actuators that have lumped dynamics, which are modelled by a set of ODE. This model structure can for example be encountered in flexible robots [3] as well as in electrical power systems [4].

In this paper, we analyse the stabilisation problem of a clamped flexible beam with actuation on a tip mass connected at the free side using a 1 dimensional (1-D) mixed PDE-ODE (m-PDE-ODE) model. This control problem has already been investigated in several research works. To cite some, in [5] this system has been studied for the force control in contact problem, and in [6] for the stabilisation of wind turbine towers with the use of disturbance observer. Further, in [7] the authors studied the problem with a nonlinear boundary controller, concluding about asymptotic stability of the closed-loop system. The exponential stabilisation of this type of system, modelled by the Euler-Bernoulli equations, has been obtained in [8] thanks to a strong dissipation term in the boundary. From a physical point of view, the strong dissipation feedback consists on the time derivative of the strain measured at the controlled side of the beam. It has been shown in [9], that a Timoshenko beam with a tip load controlled with translational and angular velocity feedback is exponentially stable in closed-loop for a time large enough.

\footnotetext{
*This work has received funding from the European Union's Horizon 2020 research and innovation programme under the Marie SklodowskaCurie grant agreement No 765579.

${ }^{1}$ Andrea Mattioni, Yongxin $\mathrm{Wu}$ and Yann Le Gorrec are with AS2M department of the FEMTO-ST research lab University of Bourgogne Franche-Compté, 24 rue Savary, 25000 Besançon, France. andrea.mattionilfemto-st.fr yongxin. wulfemto-st. fr yann. le.gorrecdens $2 m$. fr
}

To study this control problem we make use of the portHamiltonian $(\mathrm{pH})$ operator framework. The $\mathrm{pH}$ framework is dedicated to the modelling, analysis and control design of multi-physical dynamical systems [10]. In the last two decades, the $\mathrm{pH}$ system theory has been extended from lumped parameter (ODE dynamical equations) systems to distributed parameter (PDE dynamical equations) systems, starting with the theory developed in [11]. Between the different distributed $\mathrm{pH}$ formulations, we decided to use the functional analytic $\mathrm{pH}$ approach that has its roots in the seminal work [12] and that has been extended in the $\mathrm{PhD}$ thesis [13], [14] and in the monograph [15].

In this paper, we propose a control law making use of the strong dissipation feedback, that allows obtaining the exponential stability of the closed-loop system. For proving exponential stability, we use Lyapunov arguments similar to the one used in [16]. Moreover, we explicitly compute the coefficients of the exponential bounds such that to facilitate the evaluation of the performances. Finally, with the help of numerical simulations, we show that the chosen Lyapunov function is conservative, in the sense that the parameters chosen to obtain a faster exponential bound also allow to obtain a faster decrease of the system's state norm.

\section{MODELLING}

We consider a clamped flexible beam controlled at a load connected at the free side, as depicted in Fig. 1. We denote by $m_{l}$ the mass and with $I_{l}$ the moment of inertia of the load. The term $\xi \in[0, L]$ represents the beam's spatial coordinate. $w(\xi, t)$ and $\phi(\xi, t)$ represent the deflection and the rotation of a beam's cross section at a point $\xi$ and time $t$. The beam's mass density $\rho(\xi)$, the inertia mass density $I_{\rho}(\xi)$, the Young's modulus $E(\xi)$, the inertia density $I(\xi)$ and the Shear modulus $G(\xi)$ describe the space dependent characteristic parameters of the Timoshenko beam. The kinetic energy $E_{k}$ and potential energy $E_{p}$ of the system, using the Timoshenko

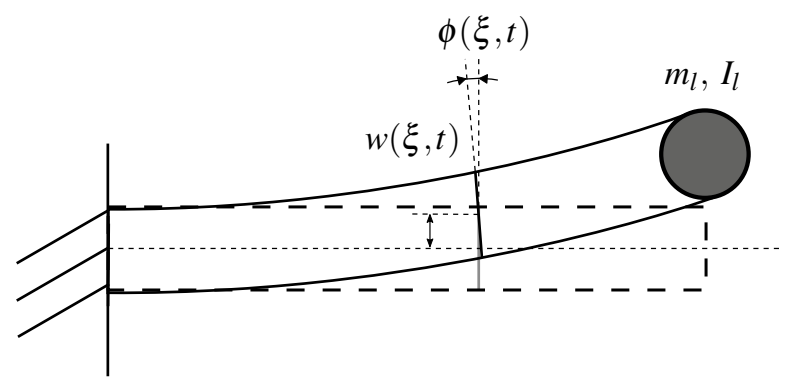

Fig. 1. Clamped flexible beam with a tip load. 
assumption, write

$$
\begin{array}{r}
E_{k}=\frac{1}{2} \int_{0}^{L}\left\{\rho(\xi) \frac{\partial w}{\partial t}(\xi, t)^{2}+I_{\rho}(\xi) \frac{\partial \phi}{\partial t}(\xi, t)^{2}\right\} d \xi \\
+\frac{1}{2} m_{l} \dot{w}(L, t)^{2}+\frac{1}{2} I_{l} \dot{\phi}(L, t)^{2} \\
E_{p}=\frac{1}{2} \int_{0}^{L}\left\{K(\xi)\left(\frac{\partial w}{\partial \xi}(\xi, t)-\phi(\xi, t)\right)^{2}\right. \\
\left.+E I(x i) \frac{\partial \phi}{\partial \xi}(\xi, t)^{2}\right\} d \xi
\end{array}
$$

where $K(\xi)=k G(\xi) A(\xi), k$ is a positive parameter depending on the beam's shape and $A(\xi)$ is the cross sectional area. In the remainder of the paper we shall not explicit the time and space dependency of the variables, unless it is not clear from the context. Considering the work of nonconservative forces as only composed by the external inputs $\delta W_{n c}=\tau \delta \phi(L, t)+f \delta w(L, t)$ and using the Principle of Least Action, it is possible to derive following dynamic equations

$$
\left\{\begin{array}{l}
\frac{\partial}{\partial t}\left(\rho \frac{\partial w}{\partial t}\right)=\frac{\partial}{\partial \xi}\left(K\left(\frac{\partial w}{\partial \xi}-\phi\right)\right) \\
\frac{\partial}{\partial t}\left(I_{\rho} \frac{\partial \phi}{\partial t}\right)=\frac{\partial}{\partial \xi}\left(E I \frac{\partial \phi}{\partial \xi}\right)+K\left(\frac{\partial w}{\partial \xi}-\phi\right) \\
I_{l} \ddot{\phi}(L, t)=-E I \frac{\partial \phi(L)}{\partial \xi}+\tau \\
m_{l} \ddot{w}(L, t)=-K\left(\frac{\partial w}{\partial z}(L)-\phi(L)\right)+f
\end{array}\right.
$$

with the additional clamping boundary conditions

$$
w(0, t)=0 \quad \phi(0, t)=0 .
$$

According to [17], we define the infinite dimensional energy variables

$$
\begin{array}{ll}
p_{t}=\rho \frac{\partial w}{\partial t} & p_{r}=I_{\rho} \frac{\partial \phi}{\partial t}(\xi, t) \\
\varepsilon_{t}=\frac{\partial w}{\partial \xi}-\phi & \varepsilon_{r}=\frac{\partial \phi}{\partial \xi}
\end{array}
$$

such to rewrite the PDE in (2) as a 1-D pH system

$$
\dot{z}=P_{1} \frac{\partial}{\partial \xi}(\mathscr{H} z)+P_{0}(\mathscr{H} z)=\mathscr{J} z
$$

where $z=\left[\begin{array}{llll}p_{t} & p_{r} & \varepsilon_{t} & \varepsilon_{r}\end{array}\right]^{T} \in Z=L_{2}\left([0, L], \mathbb{R}^{4}\right)$ and matrices defined as

$$
P_{1}=\left[\begin{array}{llll}
0 & 0 & 1 & 0 \\
0 & 0 & 0 & 1 \\
1 & 0 & 0 & 0 \\
0 & 1 & 0 & 0
\end{array}\right] \quad P_{0}=\left[\begin{array}{cccc}
0 & 0 & 0 & 0 \\
0 & 0 & 1 & 0 \\
0 & -1 & 0 & 0 \\
0 & 0 & 0 & 0
\end{array}\right] \quad \mathscr{H}=\left[\begin{array}{cccc}
\frac{1}{\rho} & 0 & 0 & 0 \\
0 & \frac{1}{I \rho} & 0 & 0 \\
0 & 0 & K & 0 \\
0 & 0 & 0 & E I
\end{array}\right] .
$$

We equip the state space $Z$ with the weighted inner-product $\left\langle z_{1}, z_{2}\right\rangle_{Z}=\left\langle z_{1}, \mathscr{H} z_{2}\right\rangle_{L_{2}}$. Therefore, the energy related to the distributed parameter part of the system can be written as $H=\frac{1}{2}\|z\|_{Z}^{2}=\frac{1}{2}\langle z, z\rangle_{Z}$. We define the boundary flow and effort according to [12]

$$
\left[\begin{array}{l}
f_{\partial}(t) \\
e_{\partial}(t)
\end{array}\right]=\frac{1}{\sqrt{2}}\left[\begin{array}{cc}
P_{1} & -P_{1} \\
I & I
\end{array}\right]\left[\begin{array}{l}
(\mathscr{H} z)(0, t) \\
(\mathscr{H} z)(L, t)
\end{array}\right] .
$$

Next, we define the boundary operators of the 1-D pH system as a selection of the previously defined boundary flow and effort

$$
\begin{aligned}
& \mathscr{B}_{1} z(t)=W_{B 1}\left[\begin{array}{l}
f_{\partial}(t) \\
e_{\partial}(t)
\end{array}\right]=\left[\begin{array}{c}
\frac{1}{\rho} p_{t}(L, t) \\
\frac{1}{I_{\rho}} p_{r}(L, t)
\end{array}\right]=u_{z, 1}(t) \\
& \mathscr{B}_{2} z(t)=W_{B 2}\left[\begin{array}{l}
f_{\partial}(t) \\
e_{\partial}(t)
\end{array}\right]=\left[\begin{array}{c}
\frac{1}{\rho} p_{t}(0, t) \\
\frac{1}{I_{\rho}} p_{r}(0, t)
\end{array}\right]=u_{z, 2}(t) \\
& \mathscr{C}_{1} z(t)=-W_{C 1}\left[\begin{array}{l}
f_{\partial}(t) \\
e_{\partial}(t)
\end{array}\right]=\left[\begin{array}{c}
K \varepsilon_{t}(L, t) \\
E I \varepsilon_{r}(L, t)
\end{array}\right]=y_{z, 1}(t) \\
& \mathscr{C}_{2} z(t)=W_{C 2}\left[\begin{array}{l}
f_{\partial}(t) \\
e_{\partial}(t)
\end{array}\right]=-\left[\begin{array}{c}
K \varepsilon_{t}(0, t) \\
E I \varepsilon_{r}(0, t)
\end{array}\right]=y_{z, 2}(t)
\end{aligned}
$$

where,

$$
\begin{aligned}
& W_{B 1}=\frac{\sqrt{2}}{2}\left[\begin{array}{cccccccc}
0 & 0 & -1 & 0 & 1 & 0 & 0 & 0 \\
0 & 0 & 0 & -1 & 0 & 1 & 0 & 0
\end{array}\right] \quad W_{B 2}=\frac{\sqrt{2}}{2}\left[\begin{array}{llllllll}
0 & 0 & 1 & 0 & 1 & 0 & 0 & 0 \\
0 & 0 & 0 & 1 & 0 & 1 & 0 & 0
\end{array}\right] \\
& W_{C 1}=\frac{\sqrt{2}}{2}\left[\begin{array}{cccccccc}
-1 & 0 & 0 & 0 & 0 & 0 & 1 & 0 \\
0 & -1 & 0 & 0 & 0 & 0 & 0 & 1
\end{array}\right] \quad W_{C 2}=-\frac{\sqrt{2}}{2}\left[\begin{array}{llllllll}
1 & 0 & 0 & 0 & 0 & 0 & 1 & 0 \\
0 & 1 & 0 & 0 & 0 & 0 & 0 & 1
\end{array}\right] \text {. }
\end{aligned}
$$

The inputs $u_{z, 1}$ and $u_{z, 2}$ represent the beam's boundary velocities at the $\xi=0$ and $\xi=L$ side, respectively. The outputs $y_{z, 1}$ and $y_{z, 2}$ describe the restoring force and torque at the $\xi=0$ and $\xi=L$ side of the beam, respectively, and are power conjugated ${ }^{1}$ to $u_{z, 1}$ and $u_{z, 2}$. According to the boundary clamping conditions (3) and the system's variable definition (4), we directly obtain that $u_{z, 2}=0$. We merge the boundary operators $(8)$ to define the complete input and output operators

$$
\begin{aligned}
& \mathscr{B} z=\left[\begin{array}{l}
\mathscr{B}_{1} z \\
\mathscr{B}_{2} z
\end{array}\right]=\left[\begin{array}{l}
W_{B 1} \\
W_{B 2}
\end{array}\right]\left[\begin{array}{l}
f_{\partial} \\
e_{\partial}
\end{array}\right]=W_{B}\left[\begin{array}{l}
f_{\partial} \\
e_{\partial}
\end{array}\right]=u_{z} \\
& \mathscr{C} z=\left[\begin{array}{l}
\mathscr{C}_{1} z \\
\mathscr{C}_{2} z
\end{array}\right]=\left[\begin{array}{l}
W_{C 1} \\
W_{C 2}
\end{array}\right]\left[\begin{array}{l}
f_{\partial} \\
e_{\partial}
\end{array}\right]=W_{C}\left[\begin{array}{l}
f_{\partial} \\
e_{\partial}
\end{array}\right]=y_{z} .
\end{aligned}
$$

Since the matrix $W_{B}$ has full rank, equation (5) together with boundary conditions (10) defines a boundary control system (see [15] page 148). Furthermore, it is possible to prove that the time derivative along trajectories of the defined distributed parameter energy equals

$$
\dot{H}=u_{z}^{T} y_{z}=u_{z, 1}^{T} y_{z, 1},
$$

where to compute the time derivative we rely on the Dini derivative concept (see Definition A.5.43 in [18]) and its calculation method (see Lemma 11.2.5 in [18]). In the following lemma we show that we have control on enough inputs of the infinite dimensional system. In the remainder of the paper we will see that this will be a key property to show the exponential stability of the closed-loop system.

Lemma 2.1: The input/output of system (5) are selected such that

$$
\|\mathscr{H} z(L, t)\|^{2}=\left\|u_{z, 1}(t)\right\|^{2}+\left\|y_{z, 1}(t)\right\|^{2} .
$$

Proof: We compute

$$
\begin{aligned}
\|\mathscr{H} z(L, t)\|^{2}= & \left(\frac{1}{\rho} p_{t}(L, t)\right)^{2}+\left(\frac{1}{I_{\rho}} p_{r}(L, t)\right)^{2} \\
& +\left(K \varepsilon_{t}(L, t)\right)^{2}+\left(E I \varepsilon_{r}(L, t)\right)^{2} \\
= & \left\|u_{z, 1}(t)\right\|^{2}+\left\|y_{z, 1}(t)\right\|^{2}
\end{aligned}
$$

that indeed prove the statement.

\footnotetext{
${ }^{1} \mathrm{~A}$ vector $u \in \mathbb{R}^{n}$ is power conjugated to a vector $y \in \mathbb{R}^{n}$ if the scalar product $\langle u, y\rangle_{\mathbb{R}^{n}}$ defines a power.
} 
The energy variables related to the finite dimensional part of the system are defined as $p_{1}=m_{l} \dot{w}(L, t)$ and $p_{2}=$ $I_{l} \dot{\phi}(L, t)$, while $u_{p}=\left[-E I \frac{\partial \phi(L)}{\partial \xi}-K\left(\frac{\partial w}{\partial z}(L)-\phi(L)\right)\right]^{T}$ depicts the restoring forces of the infinite dimensional system. Therefore we define the $\mathrm{pH}$ finite dimensional system as

$$
\left\{\begin{array}{l}
\dot{p}=u_{p}+u \\
y_{p}=M^{-1} p
\end{array}\right.
$$

where $p=\left[\begin{array}{ll}p_{1} & p_{2}\end{array}\right]^{T}, M=\operatorname{diag}\left(\left[m_{l} I_{l}\right]\right)$ is the mass matrix, $y_{p}$ represent the vector composed by the linear and angular velocity at the $\xi=L$ side of the beam and $u=[\tau f]^{T}$ is the input vector. The infinite dimensional system is power preserving interconnected with the finite dimensional system through the relations

$$
u_{z, 1}=y_{p} \quad u_{p}=-y_{z, 1}
$$

that follows from the finite and infinite energy variables definitions.

\section{CONTROL DESIGN AND EXPONENTIAL STABILITY}

The control objective consists on exponentially stabilise the origin of the system obtained by the interconnection of (5) and (14). To do so, we propose the following control law

$$
u=-R_{c} M^{-1}\left(p+K_{p} \mathscr{C}_{1} z\right)-K_{p} \frac{d}{d t}\left(\mathscr{C}_{1} z\right)
$$

where $R_{c}=\operatorname{diag}\left(\left[r_{1} r_{2}\right]\right)$ and $K_{p}=\operatorname{diag}\left(\left[k_{p, 1} k_{p, 2}\right]\right)$. The first term of the control law (16) is composed by the sum of a classical dissipation feedback and a restoring forces feedback, while the second term is known as strong dissipation feedback. Both the restoring force feedback and the strong dissipation feedback can be computed starting from strain measurements [19]. We substitute the control law (16), and the output operator $y_{z, 1}=\mathscr{C}_{1} z$ in the first equation of (14) to obtain

$$
\dot{p}=-\mathscr{C}_{1} z-R_{c} M^{-1}\left(p+K_{p} \mathscr{C}_{1} z\right)-K_{p} \frac{d}{d t}\left(\mathscr{C}_{1} z\right)
$$

that defining the new variable $\eta=p+K_{p} \mathscr{C}_{1} z$, can be rewritten as

$$
\dot{\eta}=-\mathscr{C}_{1} z-R_{c} M^{-1} \eta
$$

Defining the extended state as $x=\left[\begin{array}{c}z \\ \eta\end{array}\right] \in X=$ $L_{2}\left([0, L], \mathbb{R}^{4} \times \mathbb{R}^{2}\right.$, we can define the closed-loop operator equation

$$
\dot{x}=A x=\left[\begin{array}{cc}
\mathscr{J} & 0 \\
-\mathscr{C}_{1} z & -R_{c} M^{-1}
\end{array}\right] x
$$

with domain

$$
\begin{aligned}
\mathbf{D}(A)= & \left\{x \in X \mid(\mathscr{H} z) \in H^{1}\left([0, L], \mathbb{R}^{4}\right), \mathscr{B}_{2} z=0,\right. \\
& \left.\mathscr{B}_{1} z=M^{-1}\left(\eta-K_{p} \mathscr{C}_{1} z\right)\right\} .
\end{aligned}
$$

The closed-loop system in the new states is made by the power preserving interconnection between a infinite dimensional $\mathrm{pH}$ system with a finite dimensional $\mathrm{pH}$ system.

Since the change of variables is bounded and invertible, the equilibrium exponential stability of the system in the new coordinates (19) is equivalent to the equilibrium exponential stability of the system in the old coordinates. Moreover, the zero equilibrium in the new variables, correspond to the zero equilibrium in the old variables. In fact,

$$
p_{e q}=\eta_{e q}-K_{p} \mathscr{C}_{1} z_{e q}
$$

and if $\eta_{e q}=0$ and $z_{e q}=0$ we obtain $p_{e q}=0$. Therefore, the exponential stability of the origin in the new coordinates implies the exponential stability of the origin in the original coordinates. Using similar arguments as in Theorem 5.8 of [13], it is possible to prove the following lemma.

Lemma 3.1: The closed-loop operator $A$ with domain (20) generates a $C_{0}$-semigroup of contractions in $X$. Moreover, $A$ has compact resolvent.

Because of the previous lemma, the operator equation (19) has an unique solution that depends continuously on the initial conditions (see the $C_{0}$-semigroup Definition 5.1.2 in [15]).

In the remainder of this section we show the exponential stability of the origin of the closed-loop system (19). The proofs procedure is entirely based on the work [16]. Therefore, we skip all the proof details and we focus on the computation procedure of the exponential bound parameters for our system. To show the exponential stability, two technical lemmas giving two different estimates are needed.

Lemma 3.2: Let $x(\xi, t)$ be a solution generated by the operator $A$ with domain (20), then there exists a constant $\alpha>0$ such that the state trajectories satisfy

$$
\begin{aligned}
\left.\alpha\left(\|\mathscr{H} z(L, t)\|^{2}+\|\eta\|^{2}\right)\right) \leq \quad & y_{z, 1}^{T} K_{p} M^{-1} y_{z, 1} \\
& +\left(M^{-1} \eta\right)^{T} R_{p}\left(M^{-1} \eta\right)
\end{aligned}
$$

Proof: Use Lemma 2.1, the domain definition (20) and the norm's definition of the space $X$ to write

$$
\begin{aligned}
\|\mathscr{H} z(L, t)\|^{2}+\|\eta\|^{2} \leq & \left\|u_{z, 1}\right\|^{2}+\left\|y_{z, 1}\right\|^{2}+\|\eta\|^{2} \\
= & \left\|M^{-1}\left(\eta-K_{p} y_{z, 1}\right)\right\|^{2} \\
& +y_{z, 1}^{T} y_{z, 1}+\eta^{T} M^{-1} \eta .
\end{aligned}
$$

Then, after some computations it is possible to find

$$
\begin{aligned}
\left\|\mathscr{H}_{z}(L, t)\right\|^{2}+\|\eta\|^{2} \leq & y_{z, 1}^{T} K_{p}\left(2 K_{p} M^{-1}+K_{p}^{-1} M\right) M^{-1} y_{z, 1} \\
& +\left(M^{-1} \eta\right)^{T} R_{p} R_{p}^{-1}(2 I+M)\left(M^{-1} \eta\right)
\end{aligned}
$$

that defining $\gamma_{1}$ and $\gamma_{2}$ as the biggest eigenvalues of $2 K_{p} M^{-1}+K_{p}^{-1} M$ and $R_{p}^{-1}(2 I+M)$, respectively, we obtain

$$
\begin{aligned}
\left\|\mathscr{H}_{z}(L, t)\right\|^{2}+ & \|\eta\|^{2} \leq \gamma_{1} y_{z, 1}^{T} K_{p} M^{-1} y_{z, 1} \\
& +\gamma_{2}\left(M^{-1} \eta\right)^{T} R_{p}\left(M^{-1} \eta\right) .
\end{aligned}
$$

Finally, defining $\alpha=1 /\left(\max \left(\gamma_{1}, \gamma_{2}\right)\right)$ we obtain the inequality (22).

We give the second estimate in the following lemma.

Lemma 3.3: Let $x(\xi, t)$ be a solution generated by the operator $A$ with domain (20), then the function

$$
E(t)=\frac{1}{2} \int_{0}^{L} z(\xi, t)^{T} \mathscr{H} z(\xi, t) d \xi+\eta^{T} M^{-1} \eta
$$

is a Lyapunov function and satisfies for $t$ large enough

$$
c(t) E(x(t)) \leq \int_{0}^{t}\|\mathscr{H} z(L, \tau)\|^{2} d \tau+\int_{0}^{t}\|\eta(\tau)\|^{2} d \tau
$$


where $c(t)$ is a positive function such that $c(t) \rightarrow \infty$ for $t \rightarrow \infty$.

Proof: It is firstly necessary to remark that

$$
\begin{aligned}
\dot{E} & =\langle x, A x\rangle_{X} \\
& =-y_{z, 1}^{T} K_{p} M^{-1} y_{z, 1}-\left(M^{-1} \eta\right)^{T} R_{p}\left(M^{-1} \eta\right) \leq 0
\end{aligned}
$$

that proves that $E$ is a Lypaunov functional. It has been proven (see the Lemma 9.1.2 proof in [15]) that for a 1-D $\mathrm{pH}$ system and $\gamma, \kappa>0$ and $t>2 \gamma L$, the following inequality holds

$$
F(L) \geq F(\xi) e^{-\kappa L} \quad \text { for } \xi \in[0, L]
$$

where,

$$
F(\xi)=\int_{\gamma(L-\xi)}^{\tau-\gamma(L-\xi)} z(\xi, t)^{T} \mathscr{H} z(\xi, t) d t .
$$

Using the inequality (29) together with the fact that $E$ is a Lyapunov function, in a very similar way as did in the proof of Lemma 4.1 in [16], it is possible to prove that

$$
\begin{aligned}
& 2(t-2 \gamma L) E(x(t)) \leq \beta( \int_{0}^{t}\left\|\mathscr{H}_{z}(0, \tau)\right\|^{2} d \tau \\
&\left.+\int_{0}^{t}\|\eta(\tau)\|^{2} d \tau\right)
\end{aligned}
$$

where $\beta=\max \left\{m^{-1} L e^{\kappa L}, 1\right\}$ and $m=\min \left(\frac{1}{\rho}, \frac{1}{I_{\rho}}, K, E I\right)$. Therefore, we can conclude the prove defining $c(t)=\frac{2(t-2 \gamma L)}{\beta}$ in the last inequality.

Remark 1: According to proof of Lemma 9.1.2 in [15], $\gamma$ should be large enough to render $P_{1}^{-1}+\gamma \mathscr{H}$ and $-P_{1}^{-1}+\gamma \mathscr{H}$ positive definite, while $\kappa$ must be such that

$$
\mathscr{H}(\xi) P_{0}^{T} P_{1}^{-1}+P_{1}^{-1} P_{0} \mathscr{H}(\xi)+\frac{d \mathscr{H}}{d \xi}(\xi) \leq \kappa \mathscr{H}(\xi) .
$$

Using the matrices definition (6), the two parameters should be selected such that

$$
\begin{aligned}
& \gamma \geq \max _{\xi \in[0, L]}\left(\sqrt{\frac{\rho}{K}}, \sqrt{\frac{I_{\rho}}{E I}}\right) \\
& \kappa \geq \max _{\xi \in[0, L]}\left(\sqrt{\frac{K}{E I}}, \sqrt{\frac{\rho}{I_{\rho}}}\right) .
\end{aligned}
$$

Now, we are in position to state the theorem on exponential stability of the origin of the closed-loop operator.

Theorem 3.4: The origin of the closed-loop system (19)(20) is exponentially stable for $t$ large enough.

Proof: We use equations (28) to obtain

$$
\dot{E}(x)=-y_{z, 1}^{T} K_{p} M^{-1} y_{z, 1}-\left(M^{-1} \eta\right)^{T} R\left(M^{-1} \eta\right)
$$

and then Lemma 3.2 to write

$$
\dot{E}(x) \leq-\alpha\left(\|\mathscr{H} z(0, t)\|^{2}+\|\eta\|^{2}\right) .
$$

Integrating in time between 0 and $t$ both sides of the above equation and using Lemma (3.3), we obtain

$$
\begin{aligned}
E(x(t))-E(x(0)) \leq & -\alpha\left(\int_{0}^{t}\|\mathscr{H} z(0, \tau)\|^{2} d \tau\right. \\
& \left.+\int_{0}^{t}\|\eta(\tau)\| d \tau\right) \\
\leq & -\alpha c(t) E(x(t))
\end{aligned}
$$

which implies,

$$
E(x(t)) \leq \frac{1}{1+\alpha c(t)} E(x(0)) .
$$

Let $T(t)$ the $C_{0}$-semigroup generated by the operator $A$. The former inequality implies

$$
\|T(t)\|^{2} \leq \frac{1}{1+\alpha c(t)}
$$

Since $c(t)$ is a positive function such that $c(t) \rightarrow \infty$ for $t \rightarrow \infty$, there exists a $t^{*}>0$ such that $\|T(t)\|<1$ for all $t>t^{*}$. Consequently $w_{0}=\inf _{t>0}\left(\frac{1}{t} \log \|T(t)\|\right)<0$ and by Theorem 5.1.5 of [15] we can conclude that there exist constants $M_{w}>0$ and $w<0$ such that $\|T(t)\| \leq M_{w} e^{w t}$.

Remark 2: In the following we compute the control law parameters $K_{p}$ and $R_{p}$ such to obtain the faster decrease of the exponential bound found in Theorem 3.4. Let $T(t)$ be the $C_{0}$-semigroup generated by the closed-loop operator $A$ in (19), we recall that the exponential parameter $w_{0}$ such that $\|T(t)\| \leq M_{w} e^{w t}$ is defined as

$$
w_{0}=\inf _{t>0}\left(\frac{1}{t} \log \|T(t)\|\right),
$$

from which we understand that to obtain a small value of $w_{0},\|T(t)\|$ should be as small as possible. From inequality (38), we know that the bound of the $C_{0}$-semigroup norm $\|T(t)\|$ decreases when the $\alpha$ parameter increases. Since the function $c(t)$ does not depend on the control parameters, $K_{p}$ and $R_{p}$ should be chosen such to render $\alpha$ as big as possible. From Lemma 3.2 we know that $\alpha=1 /\left(\max \left(\gamma_{1}, \gamma_{2}\right)\right)$ and $\gamma_{1}$ and $\gamma_{2}$ are the biggest eigenvalues of $2 K_{p} M^{-1}+K_{p}^{-1} M$ and $R_{p}^{-1}(2 I+M)$, respectively. Since $K_{p}, M, R_{p}$ are all diagonal matrices, after some computations it is possible to obtain that the bigger value of $\alpha$ is reached when

$$
k_{p, i}=\frac{m_{i}}{\sqrt{2}}, \quad r_{i} \geq \frac{2+m_{i}}{2 \sqrt{2}}, \quad i=\{1,2\} .
$$

\section{NUMERICAL SIMULATIONS}

To perform numerical simulations we derive a finite element approximation of the infinite dimensional $\mathrm{pH}$ system. In particular we use the mixed finite-element discretization procedure that has been presented in [20], approximating the infinite dimensional system with 50 discretizing elements. Simulations are made in the Matlab ${ }^{\circledR}$ environment using the "ode23tb" time integration algorithm. The considered physical parameters of the clamped Timoshenko beam with tip mass are listed in Table I.

According to Remark 1 and the system's parameters, we can compute that $\gamma \geq 0.3192$ and $\kappa \geq 1$ and therefore $\beta=2.7183$. We perform different numerical simulations to highlight the system's behaviour with the choice of different control parameters. The choices are as following:

1) $K_{p}$ and $R_{p}$ are selected according to Remark 2. Therefore, $k_{p, 1}=k_{p, 2}=\sqrt{2} / 2$ and $r_{1}=r_{2}=1.5$.

2) $K_{p}$ is selected with bigger values with respect to the ones computed accordingly to Remark 2. $R_{p}$ selected 
TABLE I

SIMULATION PARAMETERS; PD CONTROLLER EXAMPLE

\begin{tabular}{ccc} 
Name & Variable & Value \\
\hline Beam's Length & $L$ & $1 \mathrm{~m}$ \\
Mass density & $\rho$ & $1 \mathrm{~kg} / \mathrm{m}^{3}$ \\
Inertia density & $I_{\rho}$ & $1 \mathrm{~kg} / \mathrm{m}$ \\
Shear parameter & $K$ & $10 \mathrm{~N} / \mathrm{m}^{2}$ \\
Young's modulus & $E$ & $10 \mathrm{~N} / \mathrm{m}^{2}$ \\
Cross section inertia & $I$ & $11 / \mathrm{m}^{2}$ \\
Tip load's mass & $I_{l}$ & $1 \mathrm{~kg} \cdot \mathrm{m}^{2}$ \\
Tip load's inertia & $I_{l}$ & $1 \mathrm{~kg} \cdot \mathrm{m}^{2}$ \\
\hline
\end{tabular}

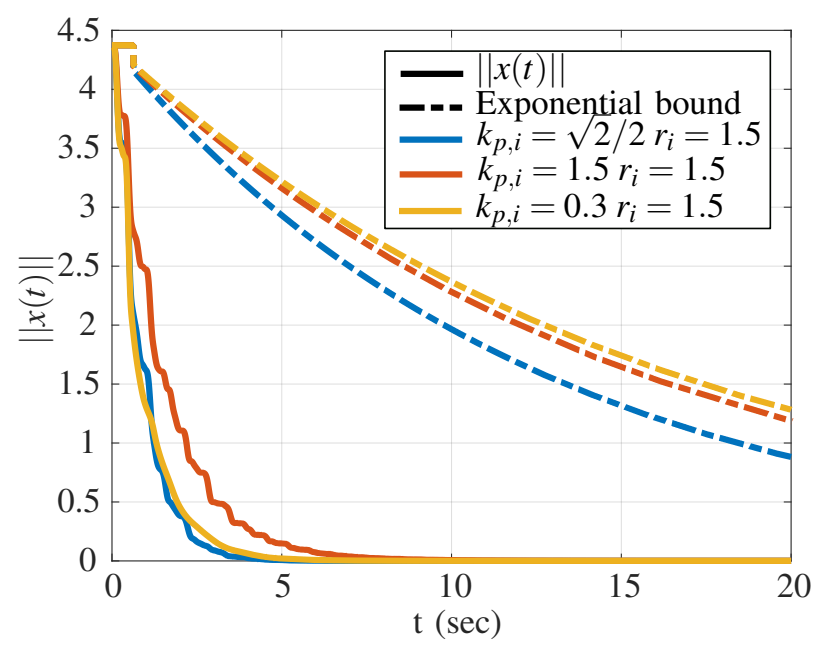

Fig. 2. Detail for $t \in[0,1]$ of the state's norm evolution along time with exponential bound.

to obtain the biggest value for $\alpha$ :

$$
r_{i} \geq \frac{2+m_{i}}{2 \frac{k_{p, i}}{m_{i}}+\frac{m_{i}}{k_{p, i}}} \quad i=\{1,2\} .
$$

Therefore we select $k_{p, 1}=k_{p, 2}=1.5$ and $r_{1}=r_{2}=1.5$.

3) $K_{p}$ is selected with smaller values with respect to the ones computed accordingly to Remark 2 and $R_{p}$ selected as in (41). Therefore, $k_{p, 1}=k_{p, 2}=0.3$ and $r_{1}=r_{2}=1.5$.

We select the initial conditions as $\varepsilon_{t}(\xi, 0)=0.5(1-$ $\left.\cos \left(\frac{2 \pi \xi}{L}\right)\right), \varepsilon_{r}(\xi, 0)=\left(1-\cos \left(\frac{2 \pi \xi}{L}\right)\right), p_{t}(\xi, 0)=p_{r}(\xi, 0)=0$ and $p_{1}=p_{2}=0$. We compute the exponential bound $w_{0}$ in (39) using a minimum search algorithm implemented in Matlab ${ }^{\circledR}$. In Fig. 3 is shown the state's norm $\|x(\xi, t)\|$ evolution along time with the three different choice of controller parameters. We can see that choosing the parameters in a way that they minimize the exponential bound leads to a controller that makes the state converge faster to the origin.

Theorem 3.4 states that the norm of the state $\|x(t)\|$ can be bounded by an exponential only for $t>2 \gamma L$. From the contraction $C_{0}$-semigroup generation of the closed-loop operator we have that $\|x(t)\| \leq\|x(0)\|$ for all $t \geq 0$. This means that the state's norm is bounded by its initial condition $\|x(t)\| \leq\|x(0)\|$ for $t \in[0,2 \gamma L]$, and by an exponential $\|x(t)\| \leq\|x(0)\| e^{w t}$ for $t>2 \gamma L$. In Fig. 2 we plot the exponential bound detail for $t \in[0,1]$ with parameters of point 1$)$.

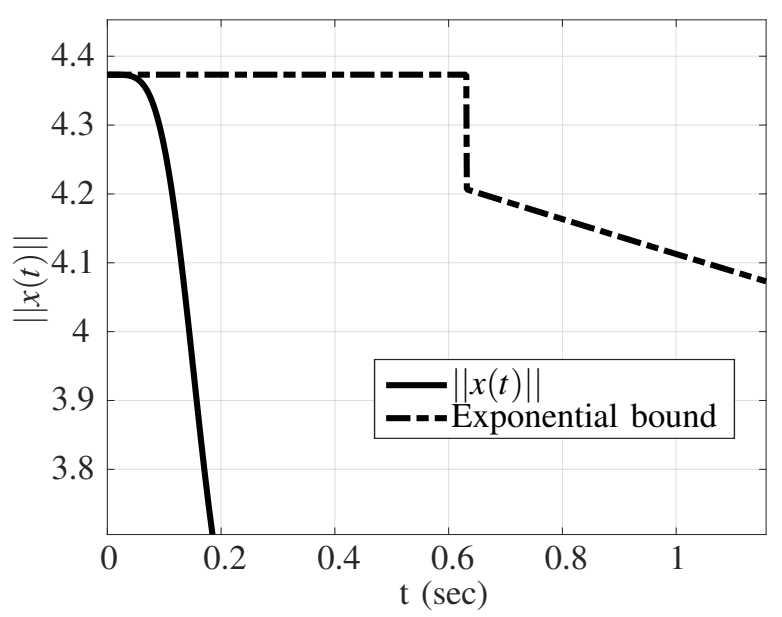

Fig. 3. State's norm evolution along time with exponential bound

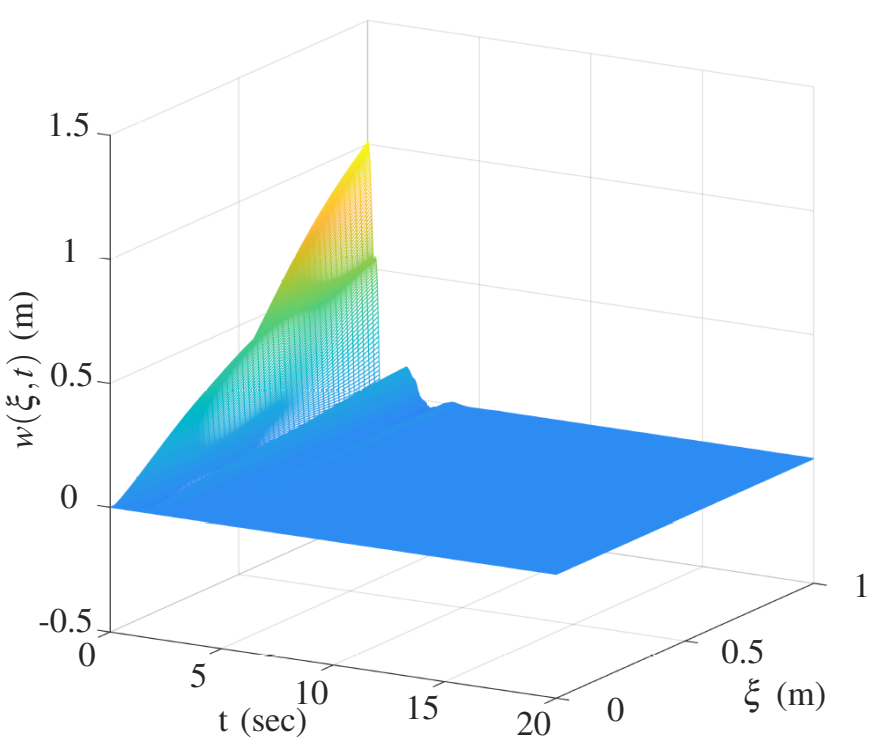

Fig. 4. Beam's deformation along time.

We can appreciate that especially for $t$ close to zero, it would be impossible to bound the state norm with an exponential. A physical explanation is that, since we are performing boundary control, we have to wait the propagation time along the whole beam length to start dissipating all the vibrations caused by the initial conditions.

In Fig. 4 is shown the beam deformation along time, where we can appreciate that the overall state converge to the origin.

\section{CONCLUSIONS AND PERSPECTIVES}

In this paper, an exponential stabilising control law has been proposed for a clamped flexible beam with actuation on a tip load connected at its free side. The control law contains a term proportional to the time derivative of the restoring forces of the flexible beam, known as strong dissipation or strain rate feedback. This term allows to "artificially" add dissipation on the boundary of the PDE modelling the flexible beam, allowing to obtain the exponential stability 
of the closed-loop system. Moreover, the parameters of the exponential bound of the state norm are explicitly given. The control parameters are computed such to obtain the faster possible exponential decrease, according to the founded parameters. Finally, numerical simulations are shown to demonstrate that the choice of parameters having a faster exponential bound allows to obtain a faster convergence of the state to the origin. The future work will focus on the generalisation on a general class of system of the proposed control law.

\section{REFERENCES}

[1] Aoues, S., Cardoso-Ribeiro, F., Matignon, D., and Alazard, D. (2019) Modeling and control of a rotating flexible spacecraft: A portHamiltonian approach. IEEE Transactions on Control Systems Technology, 27(1), 355 - 362.

[2] Boudaoud, M., Haddab, Y. and Le Gorrec, Y. (2013). Modeling and optimal force control of a nonlinear electrostatic microgripper. IEEE/ASME Transactions on Mechatronics, 18(3),1130 1139.

[3] Mattioni, A., Wu, Y. and Le Gorrec, Y. (2020). Infinite dimensional model of a double flexible-link manipulator: The port-Hamiltonian approach. Applied Mathematical Modelling, 83, 5975.

[4] Daafouz, J., Tucsnak, M. and Valein, J. (2014). Nonlinear control of a coupled PDE/ODE system modeling a switched power converter with a transmission line. Systems \& Control Letters, 70, 92 - 99.

[5] Endo, T., Sasaki, M., Matsuno, F., and Jia, Y. (2017).Contact-force control of a flexible Timoshenko arm in rigid/soft environment. IEEE Transactions on Automatic Control, 62(5), 2546 - 2553.

[6] He, W. and Ge., S. (2015). Vibration control of a nonuniform wind turbine tower via disturbance observer. IEEE/ASME Transactions on Mechatronics, 20, 237244.

[7] Miletíc, M., Stürzer, D., Arnold, A. and Kugi A. (2016). Stability of an Euler-Bernoulli beam with a nonlinear dynamic feedback system. IEEE Transactions on Automatic Control, 61(10), 27822795.

[8] Conrad, F. and Morgül, Ö. (1998). On the Stabilization of a Flexible Beam with a Tip Mass. Siam Journal on Control and Optimization, 36, 1962 - 1986.

[9] Grobbelaar-Van Dalsen, M. (2010). Uniform stability for the Timoshenko beam with tip load. Journal of Mathematical Analysis and Applications, 361, 392 - 400.

[10] Duindam, V., Macchelli, A., S. Stramigioli, S. and Bruyninckx H. eds. (2009). Modeling and Control of Complex Physical Systems The port-Hamiltonian Approach. Springer.

[11] van der Schaft, A.J. and Maschke, B.M. (2002). Hamiltonian formulation of distributed parameter systems with boundary energy flow. Journal of Geometry and Physics, 42(1), 166194.

[12] Le Gorrec, Y., Zwart, H., and Maschke, B. (2005). Dirac structures and boundary control systems associated with skew-symmetric differential operators. SIAM Journal on Control and Optimization, 44, 1864 1892.

[13] Villegas, J.A. (2007). A port-Hamiltonian approach to distributed parameter systems. Ph.D thesis Universiteit Twente.

[14] Augner, B (2018). Stabilisation of Infinite-Dimensional PortHamiltonian Systems via Dissipative Boundary Feedback. Ph.D thesis Bergische Universität Wuppertal.

[15] Jacob, B. and Zwart, H. (2012). Linear Port-Hamiltonian Systems on Infinite-dimensional Spaces. Number 223 in Operator Theory: Advances and Applications. Springer Verlag.

[16] Ramirez, H., Le Gorrec, Y., Macchelli, A., and Zwart, H. (2014). Exponential stabilization of boundary controlled port-Hamiltonian systems with dynamic feedback. IEEE Transactions on Automatic Control, 59(10), 2849 - 2855.

[17] Macchelli, A. and Melchiorri, C. (2004). Modeling and control of the Timoshenko beam: the distributed port Hamiltonian approach. SIAM Journal on Control and Optimization, 43(2), 743767.

[18] Curtain, R. and Zwart, H. (2020). Introduction to Infinite-Dimensional Linear Systems Theory, a state space approach. Springer, 1st edition.

[19] de Queiroz, M.S., Dawson, D.M. and Zhang, F. (1997). Boundary control of a rotating flexible body-beam system. Proceedings of the 1997 IEEE International Conference on Control Applications, 812 817.
[20] Golo, G., Talasila, V., van der Schaft, A., and Maschke, B. (2004). Hamiltonian discretization of boundary control systems. Automatica, $40,757-771$. 